\title{
Nonconvex Economic Dispatch Using Particle Swarm Optimization with Time Varying Operators
}

\author{
Vinay Kumar Jadoun, Nikhil Gupta, K. R. Niazi, and Anil Swarnkar \\ Malaviya National Institute of Technology, Jaipur 302017, India \\ Correspondence should be addressed to Vinay Kumar Jadoun; vjadounmnit@gmail.com
}

Received 23 May 2014; Revised 17 September 2014; Accepted 18 September 2014; Published 12 October 2014

Academic Editor: Nikos D. Lagaros

Copyright ( 2014 Vinay Kumar Jadoun et al. This is an open access article distributed under the Creative Commons Attribution License, which permits unrestricted use, distribution, and reproduction in any medium, provided the original work is properly cited.

This paper presents a particle swarm optimization (PSO) to solve hard combinatorial constrained optimization problems such as nonconvex and discontinuous economic dispatch (ED) problem of large thermal power plants. Several measures have been suggested in the control equation of the classical PSO by modifying its operators for better exploration and exploitation. The inertia operator of the PSO is modulated by introducing a new truncated sinusoidal function. The cognitive and social behaviors are dynamically controlled by suggesting new exponential constriction functions. The overall methodology effectively regulates the velocity of particles during their flight and results in substantial improvement in the classical PSO. The effectiveness of the proposed method has been tested for economic load dispatch of three standard test systems considering various operational constraints like valve-point loading effect, prohibited operating zones (POZs), network power loss, and so forth. The application results show that the proposed PSO method is very promising.

\section{Introduction}

The economic dispatch (ED) aims at determining the optimal scheduling of thermal generating units so as to minimize the fuel cost while satisfying several operational and power system network constraints. The generator fuel cost functions are invariably nonlinear and also exhibit discontinuities due to prohibited operating zones (POZs). In addition, the valvepoint loading effect causes nonconvex characteristic with multiple minima in the generator fuel cost functions and thus imposes challenges of obtaining the global optima for high dimensional ED problems. Thus, ED is a highly nonlinear, complex combinatorial, nonconvex, and multiconstraint optimization problem with continuous decision variables.

The classical mathematical methods like gradient, Lagrange relaxation methods, and so forth, except dynamic programming, are not suitable for such complex optimization problems. The modern metaheuristic search techniques such as particle swarm optimization (PSO), genetic algorithms (GAs), biogeography-based optimization (BBO), differential evolution (DE), ant colony optimization (ACO), artificial bee colony $(\mathrm{ABC})$, and hybrid swarm intelligent based harmony search algorithm (HHS) [1, 2] have shown potential to solve such complex ED problems due to their ability to obtain global or near global solution but are computationally demanding especially for modern power systems which are large and complex.

The PSO has several advantages over other metaheuristic techniques in terms of simplicity, convergence speed, and robustness [3]. It provides convergence to the global or near global optima, irrespective of the shape or discontinuities of the cost function [4]. The potential of PSO to handle nonsmooth and nonconvex ELD problem was demonstrated by [5, 6]. However, the performance of the PSO greatly depends on its parameters and it often suffers from the problems such as being trapped in local optima due to premature convergence [6], lack of efficient mechanism to treat the constraints [7], and loss of diversity and performance in optimization process [8]. PSO is a population based metaheuristic optimization technique in which the movement of the particles is governed 
by the two stochastic acceleration coefficients, that is, cognitive and social components and the inertia component [5]. In order to enhance the exploration and exploitation capabilities of PSO, the components affecting velocity of particles should be properly managed and controlled.

Several methods have been reported in the recent past to enhance the computational efficiency of the classical PSO. A constriction factor was suggested in the velocity updating equation to assure convergence of PSO [9-11]. However, the exact determination of this factor is computationally demanding. Selvakumar and Thanushkodi [12] modified cognitive behavior of the swarm by communicating with the worst particle. This method provides some additional diversity to the particle by the worst experience component, but showing poor local searching ability unless it is hybridized with certain other heuristic approaches. Roy and Ghoshal [13] proposed crazy PSO (CPSO), where the particle velocity is randomized within predefined limits. The idea was to randomize the velocity of some of the particles, referred to as "crazy particles," by applying a predefined probability of craziness to maintain the diversity for global search and better convergence. However, the value of predefined probability of craziness can only be achieved after several experimentations. Some attempts [14-18] have been made to vary the cognitive and social behavior of the swarm during the search process by dynamically controlling the acceleration coefficients within maximum and minimum bounds. Again the determination of limiting values of these acceleration coefficients is a difficult task, as it required many simulations. Coelho and Lee [19] randomized cognitive and social behavior of the swarm using chaotic sequences and Gaussian distribution, respectively. Selvakumar and Thanushkodi [20] proposed civilized swarm optimization (CSO), by combining societycivilization algorithm (SCA) with PSO to improve communication. The proposed algorithm provides clustered search that results in better exploration and exploitation of the search space but needs several experimentations to determine the optimum values of the control parameters of CSO. Efforts have also been made to suggest a new formulation of the control equation [6, 7]. Safari and Shayeghi [6] proposed iteration PSO (IPSO), where one additional velocity component pertaining to the best fitness of the current iteration is added in the control equation of the classical PSO to avoid local trap, but parameter setting is essential. Vlachogiannis and Lee [7] suggested new control equation in improved coordinated aggregation PSO (ICAPSO) for better communication among particles to enhance local search. They allowed particles to interact with its own best experience along with all other particles have better experience on aggregate basis, instead of the global best experience. However, the authors accepted that the performance of the proposed method is quite sensitive to various parameters setting and their tuning is essential. Chaotic PSO (CPSO) of [21] proposed adapted inertia weight which varies dynamically with fitness value for exploration and chaotic local search was used to determine the particle position for better exploitation. The improved PSO (IPSO) of [22] suggested chaotic inertia weight which decreases and oscillates simultaneously under the decreasing line in a chaotic manner. In this way, additional diversity

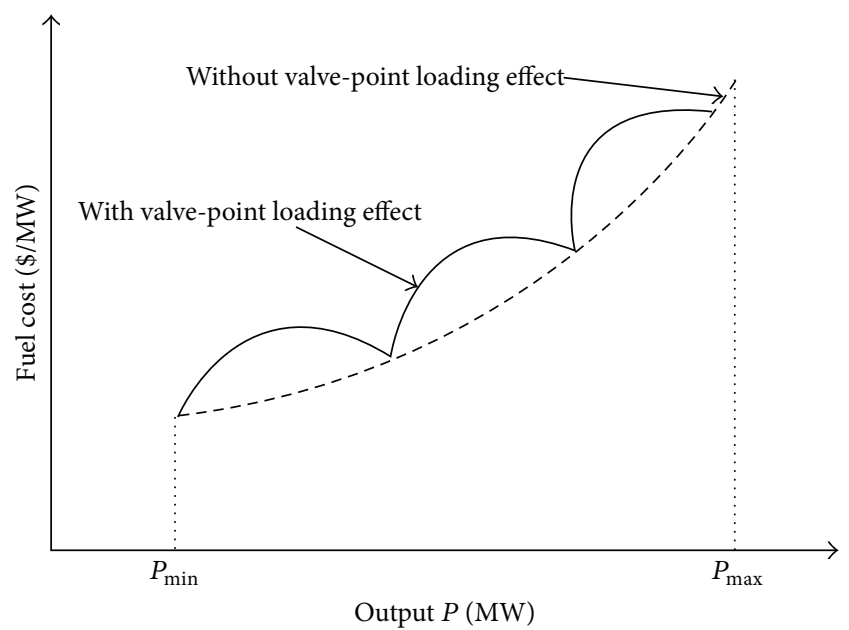

FIGURE 1: Fuel cost function with and without valve-point loading effect.

is introduced, but it requires tuning of chaotic control parameters.

This paper attempts to overcome drawbacks of some existing PSO methods and presents a modified version of PSO for economic load dispatch of power systems. Several measures have been incorporated in the control equation by modifying operators of the classical PSO by introducing new constriction functions. The proposed method effectively controls and regulates the components affecting velocity of particles so as to ensure better exploration (searching new areas) and exploitation (fine tuning of the current solution). A correction algorithm is also suggested to repair infeasible solutions whenever appeared in the computational process. The proposed method is self-adjusting and does not require experimentations to obtain the optimal values of control parameters and thus overcome the drawbacks of existing PSO methods. The effectiveness of the proposed method has been investigated on three standard test systems considering various operational constraints like valve-point loading effect, prohibited operating zones (POZs), network power loss, and so forth. The application results show that the proposed PSO method is very promising.

\section{Problem Formulation}

The generator cost function is usually considered as quadratic, when valve-point loading effects are neglected. The large turbine generators usually have a number of fuel admission valves which are operated in sequence to meet out increased generation. The opening of a valve increases the throttling losses rapidly and thus the incremental heat rate rises suddenly. This valve-point loading effect introduces ripples in the heat-rate curves which introduces nonconvexity in the generator fuel cost function as shown in Figure 1. The effect of valve-point loading effects can be modeled as sinusoidal function in the cost function. Therefore, the 
objective function for the nonconvex ED problem may be stated as

$$
\begin{aligned}
\operatorname{Minimize} F\left(P_{G i}\right)=\sum_{i=1}^{N_{G}} & \left(a_{i}+b_{i} P_{G i}+c_{i} P_{G i}^{2}\right) \\
& +\left|e_{i} \sin \left(f_{i}\left(P_{G i \min }-P_{G i}\right)\right)\right|,
\end{aligned}
$$

where $a_{i}, b_{i}$, and $c_{i}$ are the cost coefficients of the $i$ th generator, $e_{i}$ and $f_{i}$ are the valve-point effect coefficients, $P_{G i}$ is the real power output of the $i$ th generator, and $N_{G}$ is the number of generating units in the system.

Subject to the following constraints:

(1) Power Balance Constraint. The total power generation of all generators must be equal to the sum of total power demand plus the network power loss. The network power loss can be evaluated using $B$-coefficient loss formula [21, 23]. Therefore, the generator power balance equation may be stated as follows:

$$
\sum_{i=1}^{N_{G}} P_{i}=P D+\sum_{i=1}^{N_{G}} \sum_{j=1}^{N_{G}} P_{G i} B_{i j} P_{G j}+\sum_{i=1}^{N_{G}} P_{G i} B_{i 0}+B_{00},
$$

where $B_{i j}$ is the transmission loss coefficient $i=$ $1,2, \ldots, N_{G}$ and $j=1,2, \ldots, N_{G}, B_{i 0}$ is the $i$ th element of the loss coefficient vector. $B_{00}$ is the loss coefficient constant.

(2) Generator Constraint. For stable operation, power output of each generator is restricted within its minimum and maximum limits. The generator power limits are expressed as follows:

$$
P_{G i}^{\min } \leq P_{G i} \leq P_{G i}^{\max } .
$$

(3) Prohibited Operating Zones. Prohibited operating zones lead to discontinuities in the input output relation of generators. Prohibited zones divide the operating region between minimum and maximum generation limits into disjoint convex subregions [14, 20]. The generation limits for the $i$ th unit with $j$ number of prohibited zones can be expressed as follows:

$$
\begin{gathered}
P_{G i}^{\min } \leq P_{G i} \leq P_{G i, 1}^{L}, \\
P_{G i, j-1}^{U} \leq P_{G i} \leq P_{G i, j}^{L}, \\
P_{G i, N_{P Z i}}^{U} \leq P_{G i} \leq P_{G i}^{\max } ; \\
i \in\left\{1,2, \ldots, N_{G P Z}\right\}, \quad j \in\left\{2,3, \ldots, N_{P Z i}\right\},
\end{gathered}
$$

where superscripts $L$ and $U$ stand for the lower and upper limit of prohibited operating zones of generators. $N_{G P Z}$ and $N_{P Z i}$ denote the total number of generators with prohibited zones and the total number of prohibited zones for the $i$ th generator, respectively.

\section{Proposed PSO}

The classical PSO is initialized with a population of random solutions and searches for optima by updating particle positions. The velocity of the particle is influenced by the three components: initial, cognitive, and the social component. Each particle updates its previous velocity and position vectors according to the following model $[3,24,25]$ :

$$
\begin{gathered}
v_{i}^{k+1}=W v_{i}^{k}+c_{1} \times \operatorname{rand}_{1}() \times \frac{\text { pbest }_{i}-s_{i}^{k}}{\Delta t} \\
+c_{2} \times \operatorname{rand}_{2}() \times \frac{\text { gbest }_{i}-s_{i}^{k}}{\Delta t}, \\
s_{j}^{k+1}=s_{j}^{k}+v_{j}^{k+1} \times \Delta t,
\end{gathered}
$$

where $v_{i}^{k}$ is the velocity of $i$ th particle at $k$ th iteration, $\operatorname{rand}_{1}()$ and $\operatorname{rand}_{2}()$ are random numbers between 0 and $1, s_{i}^{k}$ is the position of $i$ th particle at $k$ th iteration, $c_{1}, c_{2}$ are the acceleration coefficients, pbest ${ }_{i}$ is the best position of $i$ th particle achieved based on its own experience, gbest $_{i}$ is the best particle position based on overall swarm experience, $\Delta t$ is the time step, usually set to 1 second, and $W$ is the inertia weight which is allowed to decrease linearly as follows:

$$
W=W_{\min }+\frac{\left(W_{\max }-W_{\min }\right) \times\left(\text { itr }_{\max }-\mathrm{itr}\right)}{\operatorname{itr}_{\max }}
$$

where $W_{\min }$ and $W_{\max }$ are the minimum and maximum value of inertia weight, respectively, itr max $_{\text {is }}$ is maximum number of iterations and itr is the current number of iteration.

For better performance of PSO, the particles must fly with higher velocities during the early flights to enhance global search and should be relatively slow during later flights of the journey to improve local search. Therefore, with appropriate regulation of particle's velocity during the journey, the performance of PSO could be improved. Initially, the impact of cognitive component must be high and that of the social component be less to ensure global exploration of the search space by all particles without trapping into a local minima. During later search, the impact of social component must increase and that of the cognitive component must decrease to divert all particles towards global best to improve the convergence. This is essential for a good balance between exploration and exploitation as suggested by [15].

In classical PSO, only the initial velocity component using inertia weight is regulated dynamically. However, the cognitive and social behavior of the swarm, though randomized to ensure diversity, is statically controlled by assigning constant values to acceleration coefficients. These cognitive and social components of velocity are added in the regulated initial velocity component to decide the movement of particles. This probably results in uncontrolled particle velocities during the whole computation process and thus causes insufficient exploration and exploitation of the search space. This results in poor convergence due to local trapping. Therefore, a modified control equation (7) is suggested for dynamically regulating particle's velocity during their whole course of 
the flight. The modifications suggested in the control equation are explained as follows:

$$
\begin{aligned}
v_{i}^{k+1}= & W \times v_{i}^{k}+\zeta_{1} \times C_{1 b} \times \operatorname{rand}_{1}() \times \frac{\text { pbest }_{i}-s_{i}^{k}}{\Delta t} \\
& +\left(1-\zeta_{1}\right) \times C_{1 p} \times \operatorname{rand}_{2}() \times \frac{s_{i}^{k}-\text { ppoor }_{i}}{\Delta t} \\
& +\zeta_{2} \times C_{2} \times \operatorname{rand}_{3}() \times \frac{\text { gbest }_{i}-s_{i}^{k}}{\Delta t} .
\end{aligned}
$$

In (7), the inertia weight is modified to regulate the tradeoff between the global exploration and the local exploitation of the swarm. The poor experience ppoor $r_{i}$ has been added to improve the cognitive component. Further, dynamic acceleration coefficients have been introduced using constriction functions $\zeta_{1}$ and $\zeta_{2}$ to regulate the cognitive and social behaviors of the swarm. These modifications are discussed in the following sections.

3.1. Inertia Weight Update. In [25], Shi and Eberhart suggested linear modulation of the inertia weight. This trend is followed to solve ELD problems using PSO by many researchers till date and some of them can be mentioned as $[4,6,8,12,13,15,19,20,25,26]$, and so forth. In the proposed method, the inertia weight has been allowed to vary in accordance with a truncated sinusoidal function rather than to decrease linearly. The modulations suggested to update the inertia weight is governed by the following relation:

$$
\begin{array}{r}
W=W_{\min }+\left(W_{\max }-W_{\min }\right) \cos ^{2}\left(\frac{\theta}{2}\right) ; \\
0 \leq \theta \leq \pi,
\end{array}
$$

where $\theta=X \times \operatorname{itr}+Y$ and the coefficients $X$ and $Y$ are given by (9). itr is the iteration count which is in general varied from itr $_{\min }$ to itr $_{\max }$

$$
\begin{aligned}
& X=\frac{\pi}{\left(\operatorname{itr}_{\max }-\mathrm{itr}_{\min }\right)}, \\
& Y=\frac{-\pi \times \mathrm{itr}_{\min }}{\left(\mathrm{itr}_{\max }-\mathrm{itr}_{\min }\right)} .
\end{aligned}
$$

Figure 2 shows a comparison of the conventional linear modulation and sinusoidal modulation for the inertia weight to be employed in the proposed PSO. It can be depicted from the figure that using the sinusoidal variations in the inertia weight, the inertia component of the velocity of particles maintained always higher during the early half and lower during the later half of the search, when compared with its linear variations. Therefore, using sinusoidal modulations the coarse search is enhanced during the early half by exploring larger search space with higher values assigned to particle velocities. And, during the later half, the fine search is enhanced by assigning lower values to particle velocities. This facilitates particles to explore the regions in the close proximity of near global solution.

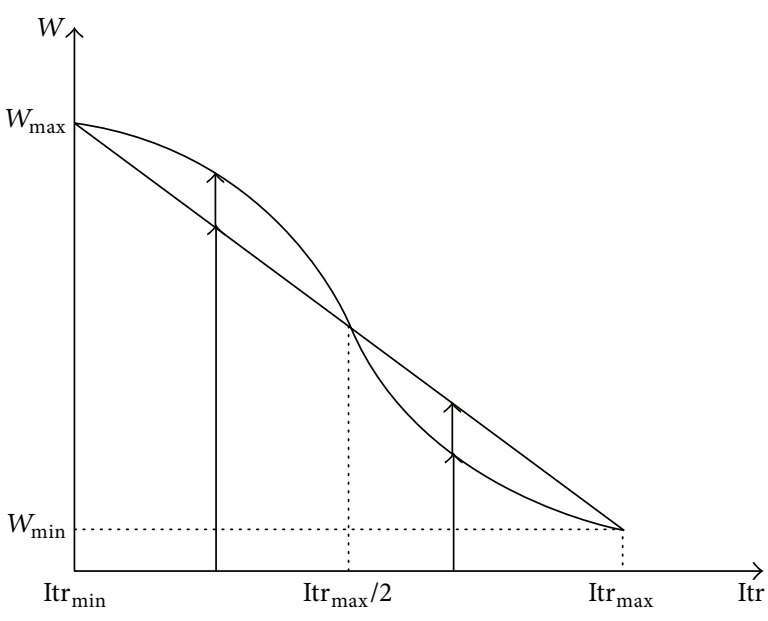

FIgURE 2: Comparison of linear and sinusoidal modulations of inertia weight.

3.2. Updating of Poor Experience. The cognitive behavior was split in [12] by considering the worst experience, in addition to the best experience, of the particle. Though this modification provides additional diversity, it still demands a local random search to enhance exploitation potential of the PSO. This occurs as the particle's velocity is not well regulated during later part of the search. Therefore, the concept of poor experience ppoor $_{i}$ is suggested, instead of the worst experience, to improve cognitive behavior of the swarm. Here the current fitness of each particle is compared with its fitness value in the preceding iteration, and if it is found less, it will be treated as the poor experience. This concept is different than that of [12], where the worst particle is determined by considering the whole past experience of the particle movement. The poor particle produces much less diversity than the worst particle and thus exploit the region near global optima, during later iterations, in much better way without the support of any local random search.

3.3. Dynamic Control of Acceleration Coefficients. In classical PSO, the cognitive and social behaviors are governed by assigning static values to acceleration coefficients. Many researchers, as discussed earlier, suggested that these acceleration coefficients must be dynamically controlled to regulate particle's velocity during the whole computation process. In the present work, the acceleration coefficients are dynamically controlled by suggesting new exponential constriction functions $\zeta_{1}$ and $\zeta_{2}$. These constriction functions dynamically regulate the cognitive and social behaviors of the swarm, thus limiting particles' velocities during their whole course of the flight and are given by

$$
\begin{aligned}
& \zeta_{1}=\mathrm{e}^{-\mu_{1} \eta}, \\
& \zeta_{2}=k \mathrm{e}^{\mu_{2} \eta},
\end{aligned}
$$




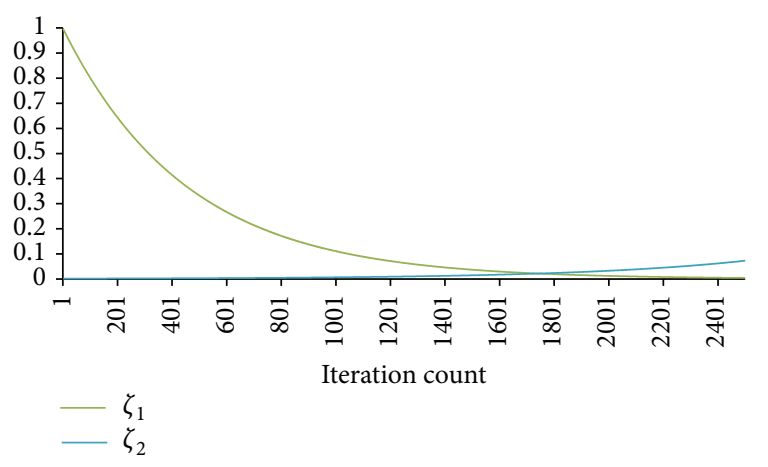

FIGURE 3: Proposed exponential constriction functions.

TABLE 1: Particle encoding for the proposed PSO.

\begin{tabular}{llllll}
\hline$P_{G 1}$ & $P_{G 2}$ & $\cdots$ & $P_{G i}$ & $\cdots$ & $P_{G N}$ \\
\hline
\end{tabular}

where

$$
\begin{gathered}
\eta=\frac{\mathrm{itr}}{\mathrm{itr} \mathrm{max}_{\max }} ; \quad \operatorname{itr}_{\min } \leq \mathrm{itr} \leq \mathrm{itr}_{\max } \\
k=\frac{\zeta_{1} C_{1 b}}{\zeta_{2} C_{2}} .
\end{gathered}
$$

The coefficient of exponent $\mu_{1}$ has been considered -5.5 , as the term $\mathrm{e}^{-\mu_{1} \eta}$ is not perceptible at the end of search. With $\mu_{2}$ as 4 , the coefficient $k$ is determined for exact match of $\zeta_{1}$ and $\zeta_{2}$ at two-third of the search. The variation in $\zeta_{1}$ and $\zeta_{2}$ with iterations is shown in Figure 3 for the above mentioned value of the exponent coefficients. It can be depicted from the figure that the dominance of cognitive behavior falls sharply and that of the social behavior rises gradually as the search progresses. Thus, during the early part of the computational search, the cognitive behavior is well dominated over the social behavior of the swarm to enhance the global search for most probable area having the global optima. However, during the later part, it is the social behavior of the swarm that dominates over its cognitive counterpart. This may enhance local exploitation by the swarm to search global or near global optima.

These alterations in the control equation of the classical PSO regulate particle velocity within predefined bounds without any additional formulation as reported in many improved versions of PSO $[4,6-8,10,13-16,18,21,26]$, yet preserving diversity due to the stochastic nature of cognitive and social behaviors of the swarm.

3.4. Particle Encoding and Initialization. The solution of an ED problem is the set of most optimal generations for the desired objective(s) bounded by certain operational constraints. In the proposed PSO, the particles are encoded in real numbers as the set of current generations in MW, as shown in Table 1.

Where $P_{G i}$ denotes generation of the $i$ th generator in $\mathrm{MW}$, the initial population is randomly created with predefined number of particles to maintain diversity. Each of these particles satisfies problem constraints defined by (2)-(4). Infeasible particle, if appeared, is not rejected but corrected using a correction algorithm as described later in the section. This improves the computational efficiency of the PSO. The fitness of each particle is evaluated using (1) and then pbest, ppoor, and gbest are initialized. The initial velocity of particles is assumed to be zero.

3.5. Correction Algorithm. The velocity and position update may create infeasible solutions. Infeasible individuals are not rejected but are corrected to feasible individuals by using a correction algorithm. For the purpose, the generations of all generators are adjusted by their respective bounded generation limits and then the error is calculated from the power balance equation. The error in the power is equally distributed among all generators and the procedure is repeated till the error is reduced to a predefined mismatch value $\epsilon$. In this work the mismatch is considered as 0.001 . This reduces the computational burden of PSO.

3.6. Elitism and Termination Criterion. In stochastic based algorithms like PSO, the solution with the best fitness in the current iteration may be lost in the next iteration. Therefore, the particle with the best fitness is kept preserved for the next iteration. The algorithm is terminated when either all particles reach to the best position or the predefined maximum iteration number is reached. The flow chart of the proposed method is shown in Figure 4.

\section{Simulation Results}

The proposed algorithm is tested on 13-generator system [23] and 40-generator system [23]. The control parameters used for all these systems to solve the ED problem using classical and proposed PSO are considered as mentioned in Table 2. The proposed algorithm has been developed using MATLAB and simulations have been carried on a personal computer of Intel i5, 3.2 GHz, and 4 GB RAM.

4.1. Case Study 1: 13-Generator System. The proposed method is applied on 13 thermal generating units which consist of valve-point effect and network power losses. The thermal generating units' data and $B$-coefficient power loss data are referred from [23]. The ED problem is solved for a power demand of $2520 \mathrm{MW}$. The simulation results obtained for the best and average fuel cost, total power output, and power losses after 100 independent trails using proposed PSO are presented in Table 3. The table shows that the proposed PSO is capable of obtaining better best, average, and best fuel costs with smaller power loss than other available existing stochastic methods in reasonable CPU time. Thus, the proposed method provides good quality solution to solve complex nonconvex ED problems. The best generating schedule obtained using the proposed PSO is presented in Appendix.

4.2. Case Study 2: 40-Generator System with Valve-Point Effect. The effectiveness of the proposed method is now investigated 


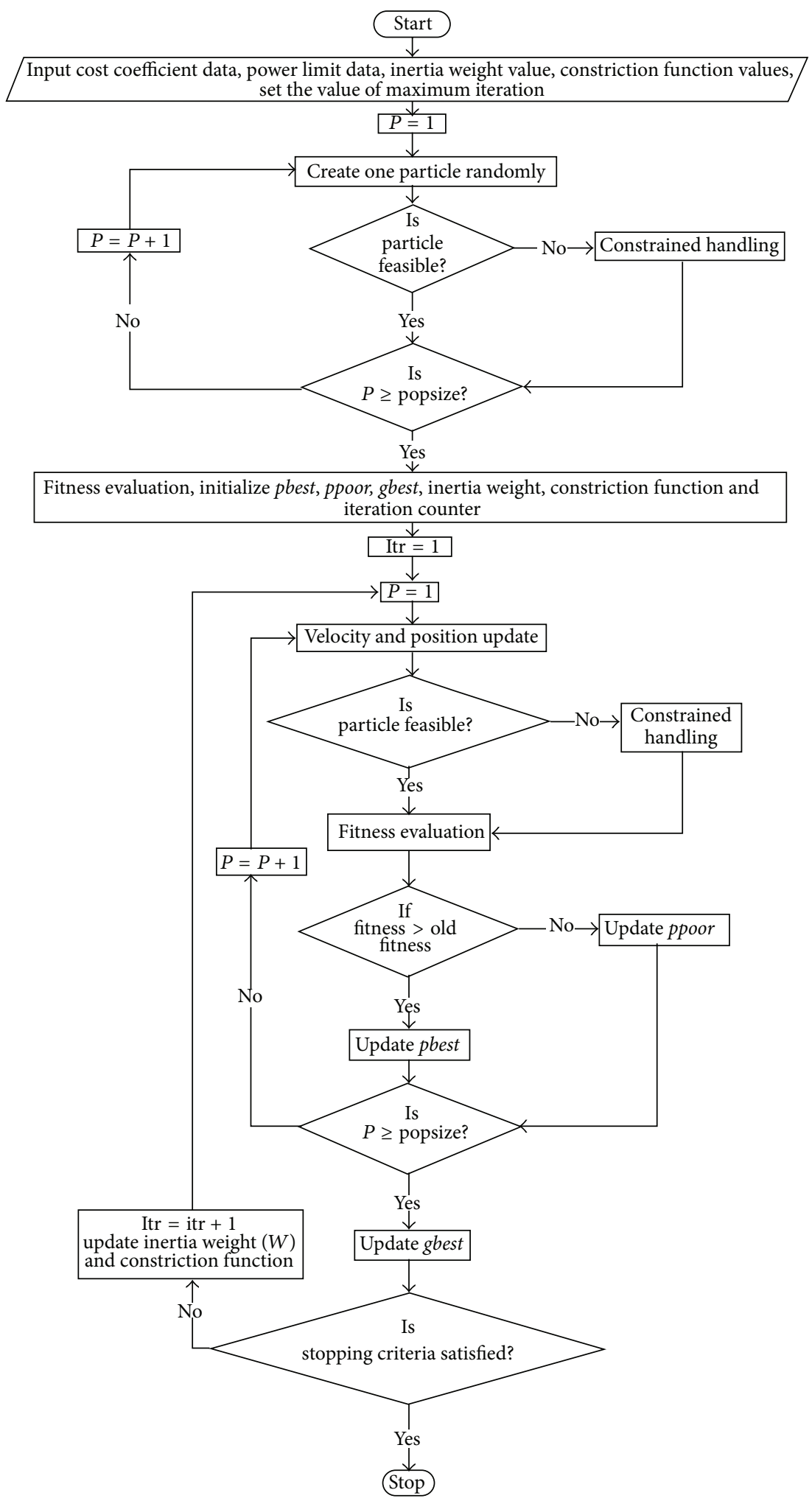

FIGURE 4: Flow chart of the proposed PSO. 
TABLE 2: Various parameters for classical PSO and proposed PSO.

\begin{tabular}{lcccccccc}
\hline Method & $W_{\min } / W_{\max }$ & $C_{1 b}$ & $C_{1 p}$ & $C_{2}$ & $\mu_{1}$ & $\mu_{2}$ & itr $_{\max }$ & Population size \\
\hline Classical PSO & $0.1 / 0.9$ & 2 & - & 2 & - & - & 2500 & 100 \\
Proposed PSO & $0.1 / 0.9$ & 1.5 & 0.5 & 2 & -5.5 & 4 & 2500 & 100 \\
\hline
\end{tabular}

TABle 3: Comparison results for case study 1.

\begin{tabular}{lcccccc}
\hline Method & $\begin{array}{c}\text { Best fuel cost } \\
(\$ / \mathrm{hr})\end{array}$ & $\begin{array}{c}\text { Average fuel cost } \\
(\$ / \mathrm{hr})\end{array}$ & $\begin{array}{c}\text { Worst fuel cost } \\
(\$ / \mathrm{hr})\end{array}$ & $\begin{array}{c}\text { Total power } \\
(\mathrm{MW})\end{array}$ & $\begin{array}{c}\text { Power loss } \\
(\mathrm{MW})\end{array}$ & $\begin{array}{c}\text { CPU time } \\
(\mathrm{s})\end{array}$ \\
\hline GA [27] & 24632.42 & 24874.93 & 25188.59 & 2559.87 & 39.87 & 2.25 \\
DE [27] & 24819.32 & 25217.64 & 25656.40 & 2562.34 & 42.34 \\
HDE [27] & 24591.76 & 24739.53 & 25074.90 & 2559.16 & 39.16 & 2.58 \\
STHDE [27] & 24560.08 & 24706.63 & 24872.44 & 2564.33 & 44.33 & 3.57 \\
ICA-PSO [7] & 24540.06 & 24561.46 & 24589.45 & 2559.05 & 39.05 & 2.98 \\
SDE [1] & 24514.88 & 24516.31 & - & 2560.43 & 40.43 & -2.5 \\
Proposed PSO & 24514.46 & 24514.58 & 24515.26 & 2558.07 & 38.07 & 2.96 \\
\hline
\end{tabular}

TABLE 4: Comparison results for case study 2.

\begin{tabular}{|c|c|c|c|c|}
\hline Method & $\begin{array}{l}\text { Best fuel cost } \\
(\$ / h r)\end{array}$ & $\begin{array}{c}\text { Average fuel cost } \\
(\$ / \mathrm{hr})\end{array}$ & $\begin{array}{c}\text { Worst fuel cost } \\
(\$ / \mathrm{hr})\end{array}$ & $\begin{array}{c}\text { CPU time } \\
(\mathrm{s})\end{array}$ \\
\hline SQP [28] & 122904.4243 & 124883.7692 & 126585.2290 & 10.80 \\
\hline EP-SQP [29] & 122323.9700 & 122379.6300 & - & 997.73 \\
\hline PSO-SQP [29] & 122094.6700 & 122245.2500 & - & 733.97 \\
\hline PSO-LRS [12] & 122035.7946 & 123382.0000 & 125740.6300 & 31.61 \\
\hline NPSO [12] & 121704.7391 & 122221.3697 & 122995.0976 & 8.23 \\
\hline NPSO-LRS [12] & 121664.4308 & 122981.5913 & 122209.3185 & 20.74 \\
\hline DEC-SQP [30] & 121741.9800 & 123367.6500 & 125397.9600 & 925.63 \\
\hline $\operatorname{DEC}(2)-S Q P(1)[28]$ & 121741.9793 & 122295.1278 & 122839.2941 & 14.26 \\
\hline $\mathrm{ACO}[31]$ & 121532.4100 & 121606.4500 & 121679.6400 & 52.45 \\
\hline FCASO [32] & 121516.4700 & 122082.5900 & - & 145.2 \\
\hline SOH-PSO [14] & 121501.1400 & 121853.5700 & 122446.3000 & - \\
\hline TSARGA [33] & 121463.0700 & 122928.3100 & 124296.5400 & 696.0 \\
\hline CPSO-SQP [34] & 121458.5400 & 122028.1600 & - & \\
\hline GA-PS-SQP [29] & 121458.0000 & 122039.0000 & - & 46.98 \\
\hline $\mathrm{ABC}[35]$ & 121441.0300 & 121995.8200 & - & 30.02 \\
\hline CCPSO [22] & 121412.5362 & 121445.3269 & 121525.4934 & 19.3 \\
\hline ICA-PSO [7] & 121422.1000 & - & - & 139.9 \\
\hline $\mathrm{DE} / \mathrm{BBO}[36]$ & 121420.8948 & - & - & 12 \\
\hline HHS [37] & 121415.5920 & 121615.8544 & - & 16.39 \\
\hline IPSO [2] & 121412.8660 & 121509.5223 & 121546.8420 & 42.89 \\
\hline NAPSO [8] & 121412.5700 & - & - & 12.7 \\
\hline CSA [38] & 121412.5355 & 121520.4106 & 121810.2538 & 3.03 \\
\hline Proposed PSO & 121412.5355 & 121432.3215 & 121564.3454 & 9.99 \\
\hline
\end{tabular}

TABle 5: Comparison results for case study 3.

\begin{tabular}{lcccc}
\hline Method & $\begin{array}{c}\text { Best fuel cost } \\
(\$ / \mathrm{hr})\end{array}$ & $\begin{array}{c}\text { Average fuel cost } \\
(\$ / \mathrm{hr})\end{array}$ & $\begin{array}{c}\text { Worst fuel Cost } \\
(\$ / \mathrm{hr})\end{array}$ & $\begin{array}{c}\text { CPU time } \\
(\mathrm{s})\end{array}$ \\
\hline PSO [8] & 124875.8523 & 125162.7011 & - & - \\
FAPSO [8] & 122261.3706 & 122471.0751 & 122597.5196 & 19.6 \\
NAPSO [8] & 121491.0662 & 121491.2756 & 121491.5261 & 12.7 \\
CSA [38] & 121487.7727 & 121611.3170 & 122162.9295 & 14.7 \\
Proposed PSO & 121487.7718 & 121511.3114 & 121753.7157 & 8.4 \\
\hline
\end{tabular}


on the most popular test generating system taken from [23]. This system consists of 40 thermal units with nonconvexity in cost function due to valve-point loading effects. The expected power demand for this test system is $10500 \mathrm{MW}$. The results obtained after 100 independent trials of the proposed PSO are presented and compared with a variety of other available existing deterministic and population based or their hybrid techniques in Table 4. The table validates the effectiveness of the proposed PSO as it generates either comparable or better best fuel cost than other several established techniques including hybrid techniques. The table also shows that the proposed PSO is less computationally demanding than many other references including some latest ones. Although, NPSO [12] and CSA [38] demand less CPU time than the proposed $\mathrm{PSO}$, but the proposed method is capable of generating better quality solution. Thus the proposed PSO is promising to solve nonconvex ED problems. The optimal dispatch of thermal generators obtained by the proposed PSO can be referred to in Appendix.

4.3. Case Study 3: 40-Generator System with Valve-Point and POZs. Finally, the effectiveness of the proposed method is investigated on the 40 generators test generating system with discontinuities in the cost function due to prohibited operating zones. The units $10-14$ have POZs as given in [8] (POZ 2). The expected power demand for this test system is $10500 \mathrm{MW}$. The results obtained after 100 trials of the proposed PSO are presented and compared with other available existing population based techniques in Table 5. The table shows that the proposed PSO is capable of generating comparable or better result in less computational time than other established available methods. The better value of average fuel cost is obtained by proposed method than other methods. This shows robustness of the proposed PSO. Thus the high dimensional nonconvex discrete ED problems can be effectively and efficiently solved using the proposed PSO. The optimal dispatch of thermal generators obtained by the proposed PSO can be referred to in Appendix.

\section{Discussion}

In order to appreciate and understand the performance of the proposed method a comparison of cognitive and social behavior of particle in PSO and the proposed PSO is shown in Figures 5 and 6 , respectively. Figure 5 shows that, in the classical PSO, the cognitive and social behaviors of particle velocity vary randomly throughout the computational process within limits of 0 to 2 . The proposed constriction functions used to guide the cognitive and social behaviors of the swarm are allowed to vary exponentially as shown in Figure 6. The lower and upper limits of these behaviors are governed by (10). However, the sum of the best and poor cognitive behavior of the swarm remains constant during the computation process. This plays an important role in providing sufficient diversity by the poor experience during the whole flight of the swarm. It can be seen from Figure 6 that, using proposed PSO, the modulations of cognitive (best), cognitive (poor), and social behaviors though randomly distributed are dynamically controlled within exponential bounds of $1.5,0.5$, and 0.15 , respectively. This constitutes a marked difference with other versions of existing PSO. Thus the particles experience entirely different cognitive and social behaviors during their flights and need no additional mechanism to bind their velocities.

Any stochastic based search technique must be designed to accomplish global exploration and tends to facilitate local exploitation. In order to investigate the effectiveness of each of these modifications, a set of convergence characteristics for the best and average fuel cost obtained during a sample trial for 40 generators system is shown in Figures 7 and 8, respectively. In Figure 7, the characteristic "a" is for the conventional PSO, "b" refers to "a" with sinusoidal modulation in inertia weight, "c" refers to " $b$ " with improved cognitive behavior due to poor experience, and "d" refers to the proposed PSO. It can be observed from the figure that the performance of the PSO is somewhat improved when inertia weight is sinusoidally modulated and is further improved with a good margin when poor experience of particles is also considered. However, these two modifications do not seem to be sufficient to exploit the promising region effectively and efficiently. This leads to premature convergence due to local trappings which can be depicted from " $\mathrm{d}$ ". In $\mathrm{d}$, the proposed constriction functions regulate particles' velocities so that they can fly more comprehensively in the search space. In fact, due to higher initial cognitive component than the social component, the proposed PSO becomes more competent to explore wider search space during the initial phase and thus identify the promising region in about 1000 iterations. However, particles move with strong communication and thus intensively exploit the region near the global optima during later part of the search owing to high values of social component. Finally, all particles converge towards the global minima as can be observed from Figure 8 . Thus, the proposed PSO provides better exploration and exploitation of the search space and produces better quality solutions. These results also highlight that the modifications suggested in the control equation of the classical PSO are very effective as it makes the proposed PSO perform much better.

The proposed method offers better exploration and exploitation of the search space because the velocity of particles is regulated throughout their flight. The movement of a sample particle in the classical PSO and the proposed PSO is illustrated in Figures 9 and 10, respectively. These figures show the traces of initial, cognitive, and social components of particle's velocity and also the overall velocity imparted to it during a sample trial.

The classical PSO searches for about 400 iterations, as shown in Figure 9. After this, all the three components of particle's velocity became insignificant and thus the particle gets trapped into local minima. Figure 10(b) shows the cognitive component for the best experience which is then superimposed by its poor experience as in Figure 10(c) to obtain the overall cognitive component as in Figure 10(d). It can be concluded from Figure 10(d) that the poor experience is contributing to tune the cognitive behavior of the swarm. The social component, as shown in Figure 10(e), is providing 


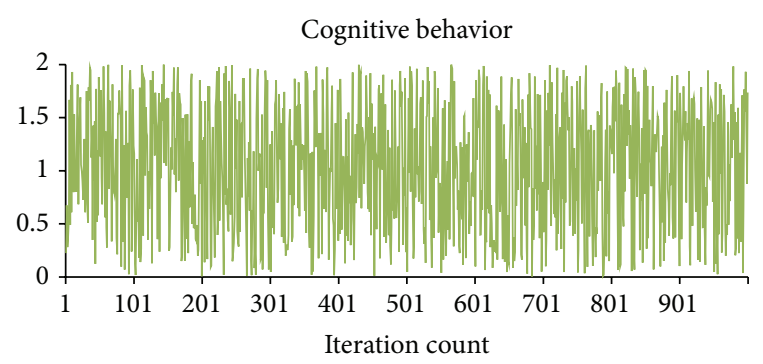

(a)

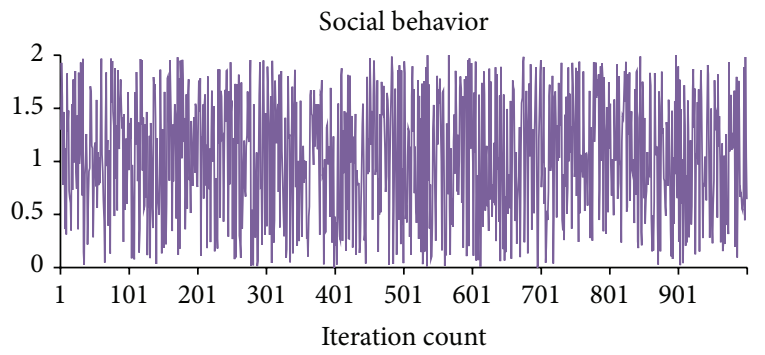

(b)

FIGURE 5: (a) Cognitive behavior and (b) social behavior in classical PSO.

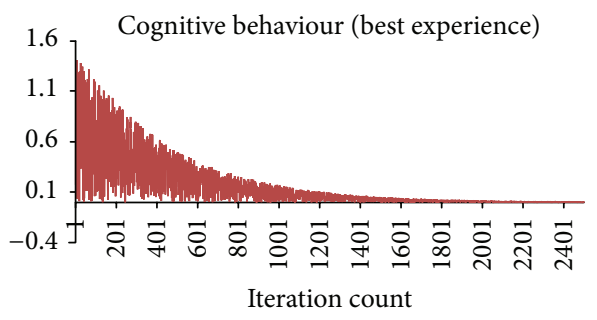

(a)

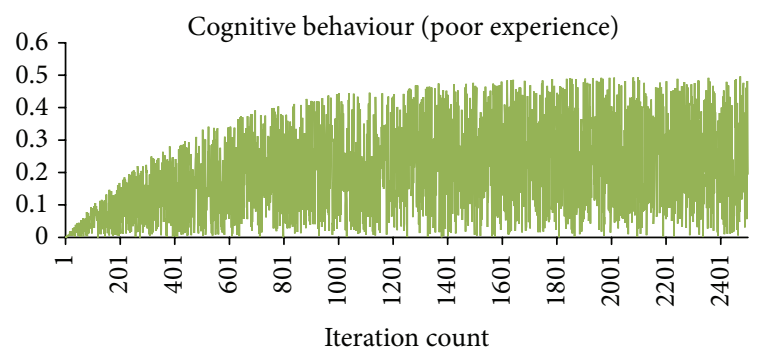

(b)

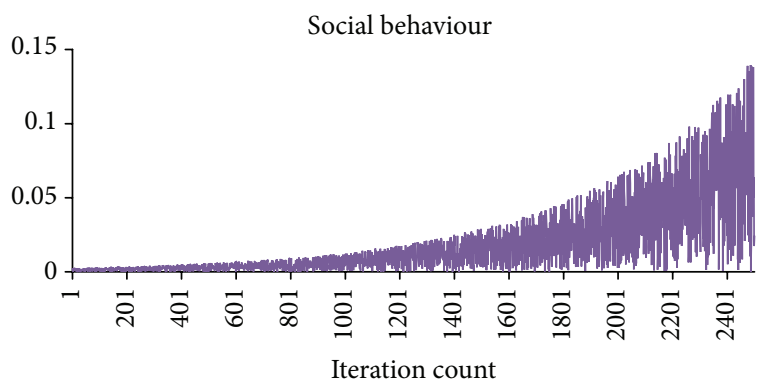

(c)

Figure 6: (a) Cognitive behavior (best experience), (b) cognitive behavior (poor experience), and (c) social behavior in proposed PSO.

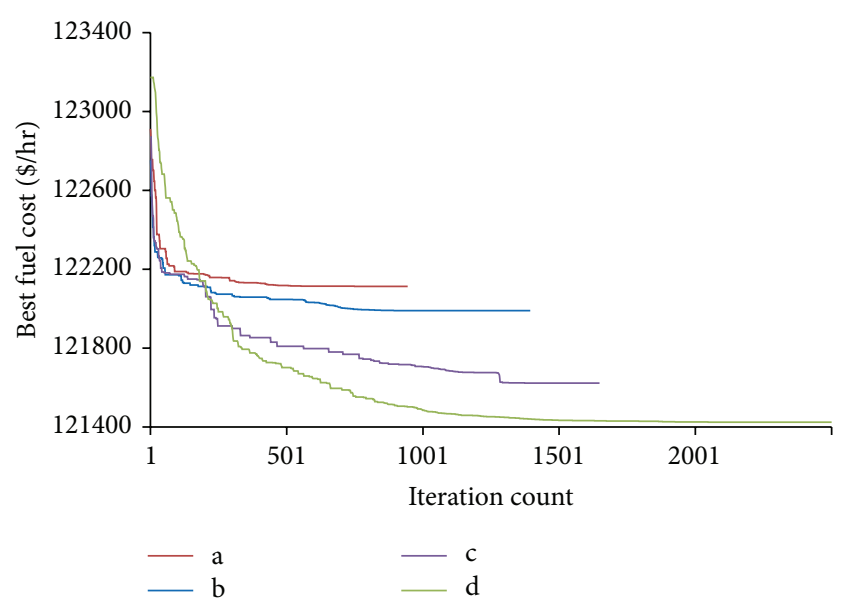

FIGURE 7: Effect on the convergence for best fuel cost by suggested modifications in the proposed PSO.

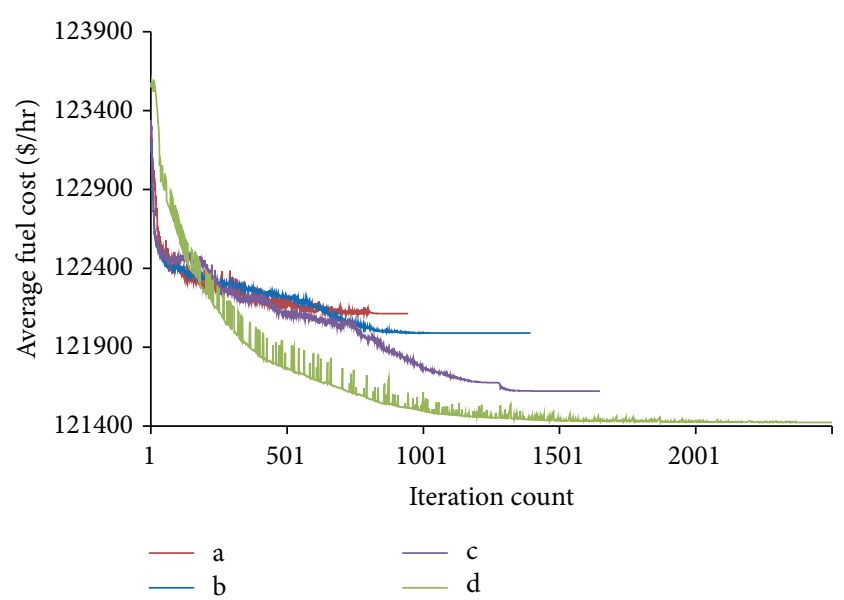

FIGURE 8: Effect on the convergence for average fuel cost by suggested modifications in the proposed PSO.

fine tuning as desired in high dimensional optimization problem. It should be noted that the social component has been kept quite weak in this work, as compared to other published literature till date and is one of the keys to obtain high quality solutions. In addition, the proposed modulation in inertia weight intends particles for better exploration and exploitation of the search space by imparting suitable velocity during the flight, as seen from Figure 10(a). The impact of improved initial, cognitive, and social components of particle's velocity is shown in Figure 10(f). The figure shows a marked improvement in particle movement during the whole computation while compared with Figure 9(d). In the proposed PSO, during early part of the search, the particles widely travelled in the search space yet their velocity is regulated by the poor experience as the social component is almost negligible. This facilitates the swarm to explore the region of global optima. However, in later part of the search, both poor and the social components are driving the swarm toward the global optima, as the cognitive best experience has been made quite weak during this part of the search. 


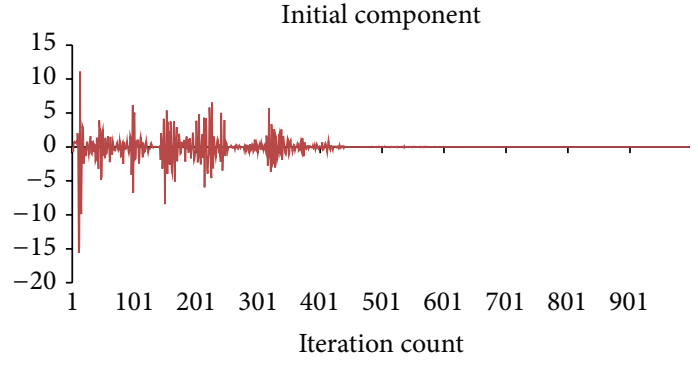

(a)

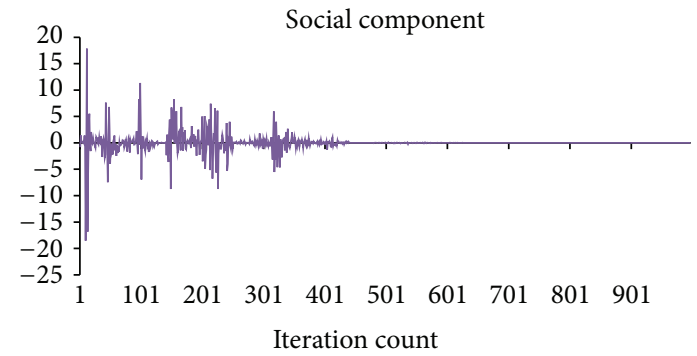

(c)

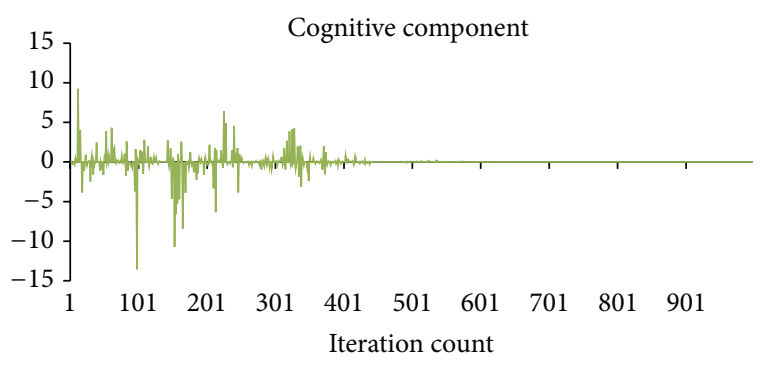

(b)

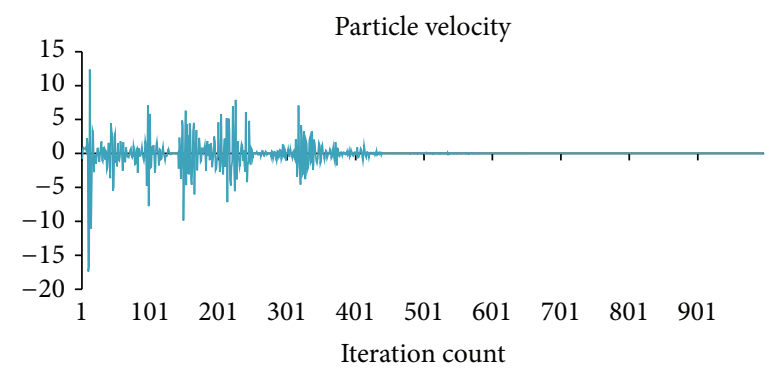

(d)

FIGURE 9: Particle velocity and its components in PSO.

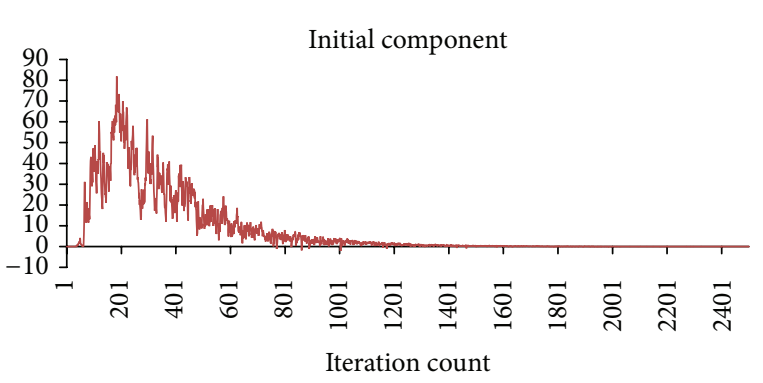

(a)

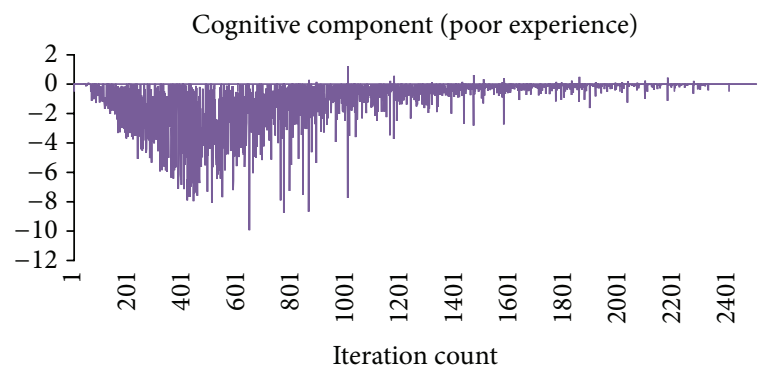

(c)

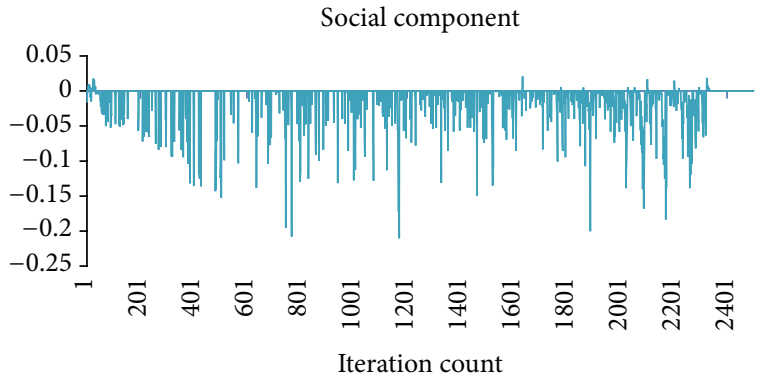

(e)

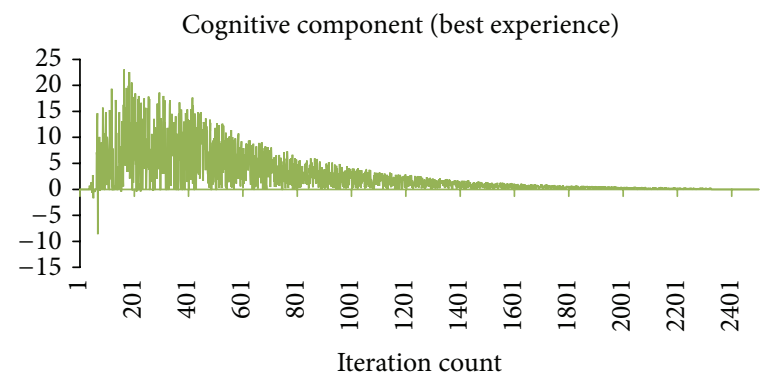

(b)

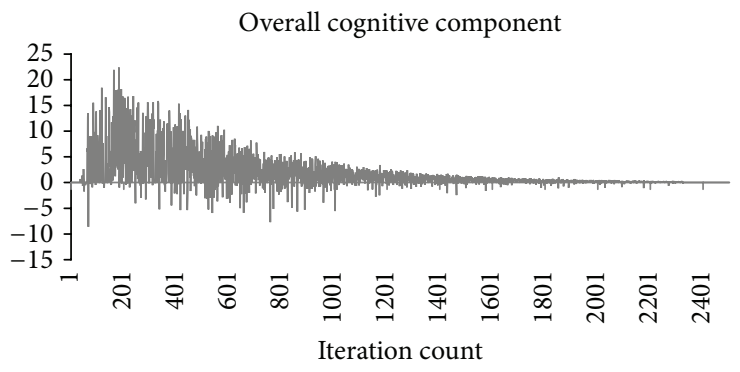

(d)

Particle velocity

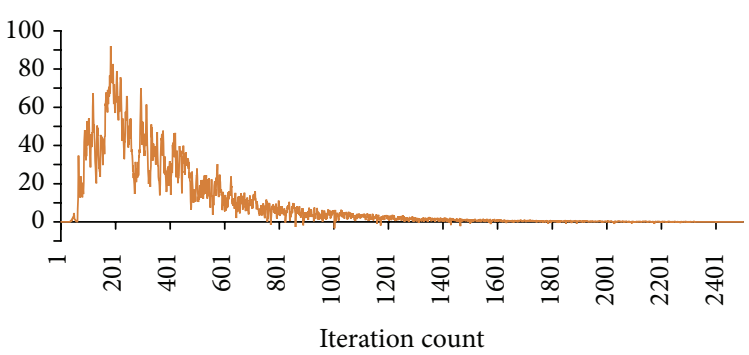

(f)

Figure 10: Particle velocity and its components in the proposed PSO. 
This improves exploitation potential of the PSO for local search. Thus, the proposed PSO provides better exploration and exploitation of the search space and thus produces better quality solutions than the classical PSO or other existing stochastic based methods.

\section{Conclusions}

The economic dispatch is a highly complex combinatorial constrained optimization problem with continuous decision variables. The classical PSO has proven potential to solve such hard combinatorial constraints optimization problem, but it usually gets trapped into local minima while dealing with high dimensional ED problems. This paper presents a modified version of PSO to make it suitable for solving highly complex ED problems. The proposed method has been tested to solve ED problems of three different test systems of different dimensions with a variety of operational and network constraints. The application results are also compared with available existing PSO methods. The application results show that the proposed method is efficient and is usually not trapped in local minima. The comparison shows that proposed method is capable of giving better results than the existing PSO and other stochastic based methods. This may be due to the fact that proposed PSO essentially aims to regulate particle velocity during its whole course of flight in such a fashion so as to enhance exploration and exploitation potentials of the PSO. The operators in the proposed PSO are made to vary dynamically by introducing new truncated sinusoidal and exponential functions. The concept of poor particle is introduced to improve the cognitive behavior of the swarm and also maintain a good balance between cognitive and social behavior of the swarm during the whole course of the flight. These modifications guide the swarm to identify the area where the global optima may exist. Thereafter, particles have suitable velocities to wandering within in this area to explore global or near global solution. Further, it has been observed that in the proposed PSO, the particle is accelerated more comprehensively during whole of its flight than in the classical PSO. This causes better exploration of the search space during the early part and better exploitation during the later part of the search. It is noteworthy that the proposed PSO is free from any mechanism to avoid local trapping and does not require any empirical formula to bound particle's velocity. Moreover, the proposed algorithm is robust as it generates better quality solutions irrespective of the initial position of the particles. The proposed PSO can be extended to solve ED problems with the inclusion of more objectives and constraints like environmental issues, reserve capacity, network security, network congestion management, and so forth.

\section{Appendix}

See Table 6.
TABLE 6: Optimal generating schedule for case studies 1, 2, and 3.

\begin{tabular}{|c|c|c|c|}
\hline \multirow{2}{*}{ Unit } & Case study 1 & Case study 2 & Case study 3 \\
\hline & Power (MW) & Power (MW) & Power (MW) \\
\hline 1 & 628.3185 & 110.799825 & 110.799789 \\
\hline 2 & 298.8000 & 110.799825 & 110.799807 \\
\hline 3 & 298.8000 & 97.3999130 & 97.3998080 \\
\hline 4 & 159.7400 & 179.733100 & 179.733093 \\
\hline 5 & 159.7400 & 87.7999050 & 87.7998250 \\
\hline 6 & 159.7400 & 140.000000 & 140.000000 \\
\hline 7 & 159.7400 & 259.599650 & 259.599600 \\
\hline 8 & 159.7300 & 284.599650 & 284.599496 \\
\hline 9 & 159.7400 & 284.599650 & 284.599700 \\
\hline 10 & 76.20000 & 130.000000 & 130.000000 \\
\hline 11 & 113.3200 & 94.0000000 & 168.798140 \\
\hline 12 & 92.10000 & 94.0000000 & 168.041419 \\
\hline 13 & 92.10000 & 214.759790 & 125.000000 \\
\hline 14 & - & 394.279370 & 400.000000 \\
\hline 15 & - & 394.279370 & 394.279018 \\
\hline 16 & - & 394.279370 & 394.279205 \\
\hline 17 & - & 489.279370 & 489.279397 \\
\hline 18 & - & 489.279370 & 489.279380 \\
\hline 19 & - & 511.279370 & 511.279377 \\
\hline 20 & - & 511.279370 & 511.279299 \\
\hline 21 & - & 523.279370 & 523.279354 \\
\hline 22 & - & 523.279370 & 523.279373 \\
\hline 23 & - & 523.279370 & 523.279372 \\
\hline 24 & - & 523.279370 & 523.279365 \\
\hline 25 & - & 523.279369 & 523.279377 \\
\hline 26 & - & 523.279370 & 523.279400 \\
\hline 27 & - & 10.0000000 & 10.0000000 \\
\hline 28 & - & 10.0000000 & 10.0000000 \\
\hline 29 & - & 10.0000000 & 10.0000000 \\
\hline 30 & - & 87.799902 & 87.7998910 \\
\hline 31 & - & 190.000000 & 190.000000 \\
\hline 32 & - & 190.000000 & 190.000000 \\
\hline 33 & - & 190.000000 & 190.000000 \\
\hline 34 & - & 164.799825 & 164.799766 \\
\hline 35 & - & 194.397782 & 164.799800 \\
\hline 36 & - & 200.000000 & 164.799803 \\
\hline 37 & - & 110.000000 & 110.000000 \\
\hline 38 & - & 110.000000 & 110.000000 \\
\hline 39 & - & 110.000000 & 109.998798 \\
\hline 40 & - & 511.279370 & 511.279348 \\
\hline
\end{tabular}

\section{Conflict of Interests}

The authors declare that there is no conflict of interests regarding the publication of this paper.

\section{Acknowledgment}

The authors would like to thank the editor and reviewers for their valuable comments and recommendations. 


\section{References}

[1] A. Srinivasa Reddy and K. Vaisakh, "Shuffled differential evolution for large scale economic dispatch," Electric Power Systems Research, vol. 96, pp. 237-245, 2013.

[2] A. K. Barisal, "Dynamic search space squeezing strategy based intelligent algorithm solutions to economic dispatch with multiple fuels," International Journal of Electrical Power \& Energy Systems, vol. 45, no. 1, pp. 50-59, 2013.

[3] J. Kennedy and R. Eberhart, Swarm Intelligence, Morgan Kaufmann, 2001.

[4] D. N. Jeyakumar, T. Jayabarathi, and T. Raghunathan, "Particle swarm optimization for various types of economic dispatch problems," International Journal of Electrical Power and Energy Systems, vol. 28, no. 1, pp. 36-42, 2006.

[5] A. Mahor, V. Prasad, and S. Rangnekar, "Economic dispatch using particle swarm optimization: a review," Renewable and Sustainable Energy Reviews, vol. 13, no. 8, pp. 2134-2141, 2009.

[6] A. Safari and H. Shayeghi, "Iteration particle swarm optimization procedure for economic load dispatch with generator constraints," Expert Systems with Applications, vol. 38, no. 5, pp. 6043-6048, 2011.

[7] J. G. Vlachogiannis and K. Y. Lee, "Economic load dispatcha comparative study on heuristic optimization techniques with an improved coordinated aggregation-based PSO," IEEE Transactions on Power Systems, vol. 24, no. 2, pp. 991-1001, 2009.

[8] T. Niknam, H. D. Mojarrad, and H. Z. Meymand, "Non-smooth economic dispatch computation by fuzzy and self adaptive particle swarm optimization," Applied Soft Computing Journal, vol. 11, no. 2, pp. 2805-2817, 2011.

[9] B. Yu, X. Yuan, and J. Wang, "Short-term hydro-thermal scheduling using particle swarm optimization method," Energy Conversion and Management, vol. 48, no. 7, pp. 1902-1908, 2007.

[10] G. Baskar and M. R. Mohan, "Security constrained economic load dispatch using improved particle swarm optimization suitable for utility system," International Journal of Electrical Power and Energy Systems, vol. 30, no. 10, pp. 609-613, 2008.

[11] L. Wang and C. Singh, "Stochastic economic emission load dispatch through a modified particle swarm optimization algorithm," Electric Power Systems Research, vol. 78, no. 8, pp. 14661476, 2008.

[12] A. I. Selvakumar and K. Thanushkodi, "A new particle swarm optimization solution to nonconvex economic dispatch problems," IEEE Transactions on Power Systems, vol. 22, no. 1, pp. 42-51, 2007.

[13] R. Roy and S. P. Ghoshal, "A novel crazy swarm optimized economic load dispatch for various types of cost functions," International Journal of Electrical Power \& Energy Systems, vol. 30, no. 4, pp. 242-253, 2008.

[14] K. T. Chaturvedi, M. Pandit, and L. Srivastava, "Self-organizing hierarchical particle swarm optimization for nonconvex economic dispatch," IEEE Transactions on Power Systems, vol. 23, no. 3, pp. 1079-1087, 2008.

[15] K. T. Chaturvedi, M. Pandit, and L. Srivastava, "Particle swarm optimization with time varying acceleration coefficients for non-convex economic power dispatch," International Journal of Electrical Power and Energy Systems, vol. 31, no. 6, pp. 249-257, 2009.

[16] K. K. Mandal and N. Chakraborty, "Daily combined economic emission scheduling of hydrothermal systems with cascaded reservoirs using self organizing hierarchical particle swarm optimization technique," Expert Systems with Applications, vol. 39, no. 3, pp. 3438-3445, 2012.

[17] Y. Wang, J. Zhou, C. Zhou, Y. Wang, H. Qin, and Y. Lu, "An improved self-adaptive PSO technique for short-term hydrothermal scheduling," Expert Systems with Applications, vol. 39, no. 3, pp. 2288-2295, 2012.

[18] B. Mohammadi-Ivatloo, "Combined heat and power economic dispatch problem solution using particle swarm optimization with time varying acceleration coefficients," Electric Power Systems Research, vol. 95, pp. 9-18, 2013.

[19] L. D. S. Coelho and C.-S. Lee, "Solving economic load dispatch problems in power systems using chaotic and Gaussian particle swarm optimization approaches," International Journal of Electrical Power and Energy Systems, vol. 30, no. 5, pp. 297-307, 2008.

[20] A. I. Selvakumar and K. Thanushkodi, "Optimization using civilized swarm: solution to economic dispatch with multiple minima," Electric Power Systems Research, vol. 79, no. 1, pp. 816, 2009.

[21] J. Cai, X. Ma, L. Li, and P. Haipeng, "Chaotic particle swarm optimization for economic dispatch considering the generator constraints," Energy Conversion and Management, vol. 48, no. 2, pp. 645-653, 2007.

[22] J.-B. Park, Y.-W. Jeong, J.-R. Shin, and K. Y. Lee, “An improved particle swarm optimization for nonconvex economic dispatch problems," IEEE Transactions on Power Systems, vol. 25, no. 1, pp. 156-166, 2010.

[23] N. Sinha, R. Chakrabarti, and P. K. Chattopadhyay, "Evolutionary programming techniques for economic load dispatch," IEEE Transactions on Evolutionary Computation, vol. 7, no. 1, pp. 8394, 2003.

[24] J. Kennedy and R. Eberhart, "Particle swarm optimization," in Proceedings of the IEEE International Conference on Neural Networks (ICNN '95), pp. 1942-1948, December 1995.

[25] Y. Shi and R. C. Eberhart, "Empirical study of particle swarm optimization," in Proceedings of the Congress on Evolutionary Computation (CEC '99), pp. 1945-1950, Piscataway, NJ, USA, July 1999.

[26] Z.-L. Gaing, "Particle swarm optimization to solving the economic dispatch considering the generator constraints," IEEE Transactions on Power Systems, vol. 18, no. 3, pp. 1187-1195, 2003.

[27] S. K. Wang, J. P. Chiou, and C. W. Liu, "Non-smooth/nonconvex economic dispatch by a novel hybrid differential evolution algorithm," IET Generation, Transmission and Distribution, vol. 1, no. 5, pp. 793-803, 2007.

[28] L. dos Santos Coelho and V. C. Mariani, "Combining of chaotic differential evolution and quadratic programming for economic dispatch optimization with valve-point effect," IEEE Transactions on Power Systems, vol. 21, no. 2, pp. 989-996, 2006.

[29] J. S. Alsumait, J. K. Sykulski, and A. K. Al-Othman, "A hybrid GA-PS-SQP method to solve power system valve-point economic dispatch problems," Applied Energy, vol. 87, no. 5, pp. 1773-1781, 2010.

[30] P. Subbaraj, R. Rengaraj, and S. Salivahanan, "Enhancement of Self-adaptive real-coded genetic algorithm using Taguchi method for Economic dispatch problem," Applied Soft Computing Journal, vol. 11, no. 1, pp. 83-92, 2011.

[31] S. Pothiya, I. Ngamroo, and W. Kongprawechnon, "Ant colony optimisation for economic dispatch problem with non-smooth cost functions," International Journal of Electrical Power and Energy Systems, vol. 32, no. 5, pp. 478-487, 2010. 
[32] J. Cai, Q. Li, L. Li, H. Peng, and Y. Yang, "A fuzzy adaptive chaotic ant swarm optimization for economic dispatch," International Journal of Electrical Power \& Energy Systems, vol. 34, no. 1, pp. 154-160, 2012.

[33] P. Subbaraj, R. Rengaraj, and S. Salivahanan, "Enhancement of self-adaptive real-coded genetic algorithm using Taguchi method for economic dispatch problem," Applied Soft Computing Journal, vol. 11, no. 1, pp. 83-92, 2011.

[34] J. Cai, Q. Li, L. Li, H. Peng, and Y. Yang, "A hybrid CPSOSQP method for economic dispatch considering the valve-point effects," Energy Conversion and Management, vol. 53, no. 1, pp. 175-181, 2012.

[35] S. Hemamalini and S. P. Simon, "Artificial bee colony algorithm for economic load dispatch problem with non-smooth cost functions," Electric Power Components and Systems, vol. 38, no. 7, pp. 786-803, 2010.

[36] A. Bhattacharya and P. K. Chattopadhyay, "Hybrid differential evolution with biogeography-based optimization for solution of economic load dispatch," IEEE Transactions on Power Systems, vol. 25, no. 4, pp. 1955-1964, 2010.

[37] V. R. Pandi, B. K. Panigrahi, R. C. Bansal, S. Das, and A. Mohapatra, "Economic load dispatch using hybrid swarm intelligence based harmony search algorithm," Electric Power Components and Systems, vol. 39, no. 8, pp. 751-767, 2011.

[38] D. N. Vo, P. Schegner, and W. Ongsakul, "Cuckoo search algorithm for non-convex economic dispatch," IET Generation, Transmission and Distribution, vol. 7, no. 6, pp. 645-654, 2013. 

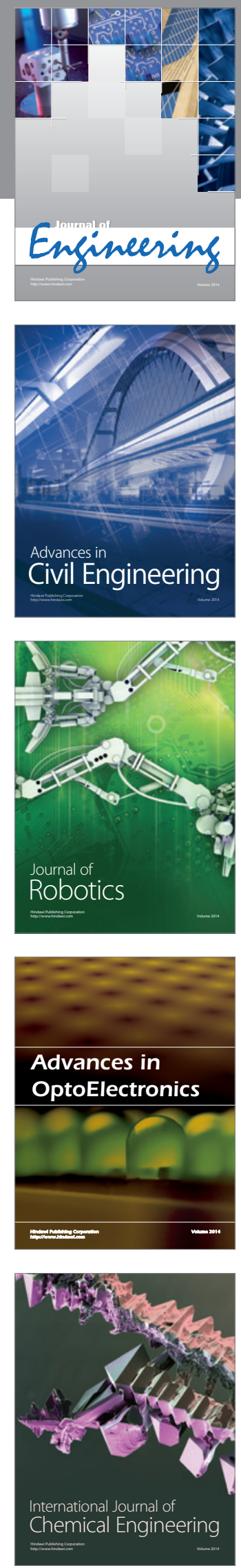

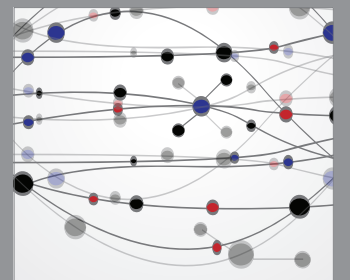

The Scientific World Journal
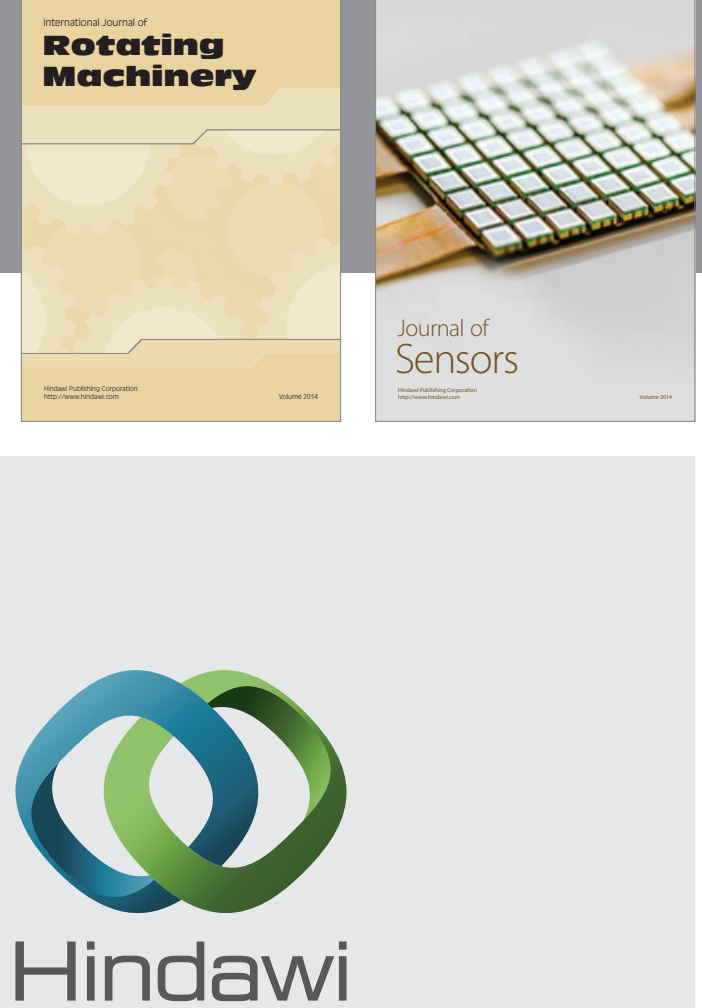

Submit your manuscripts at http://www.hindawi.com
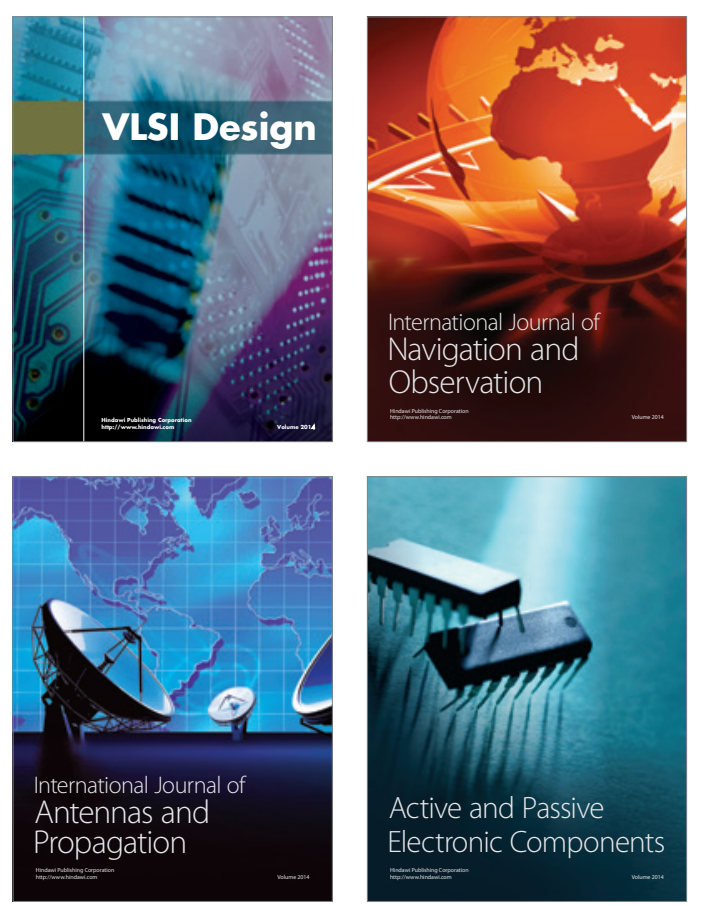
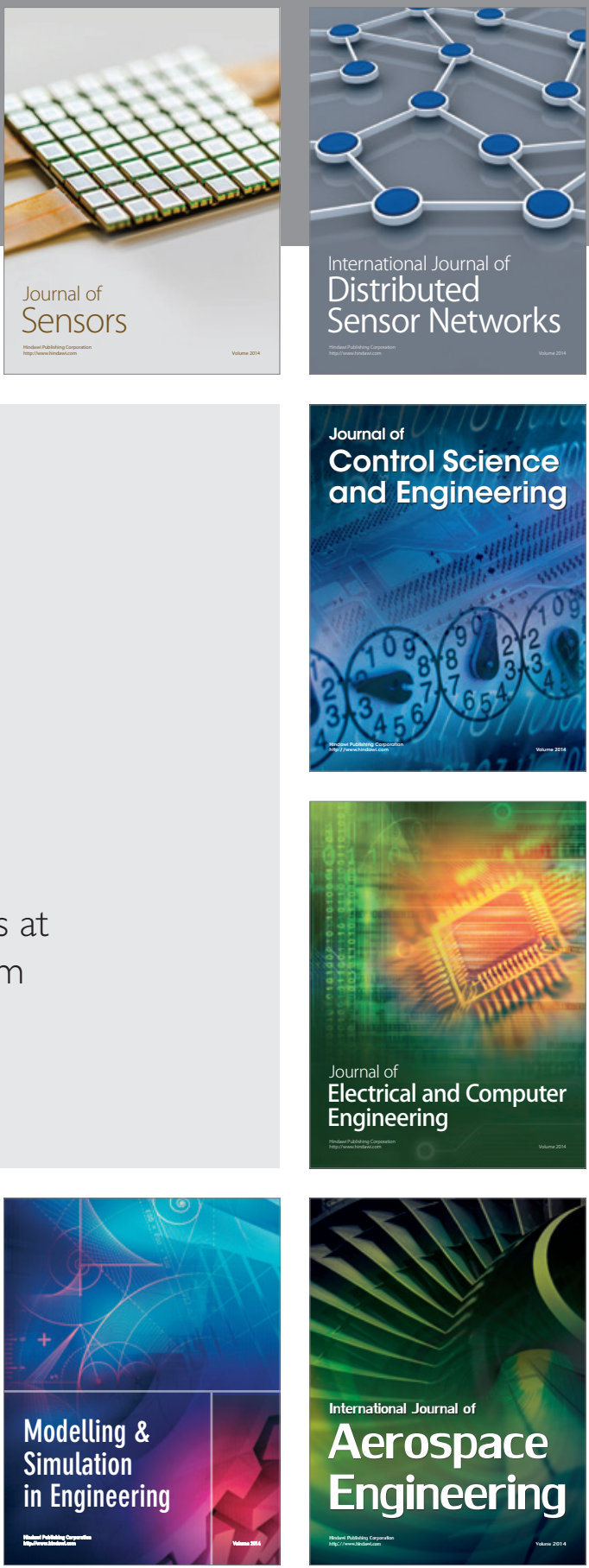

Journal of

Control Science

and Engineering
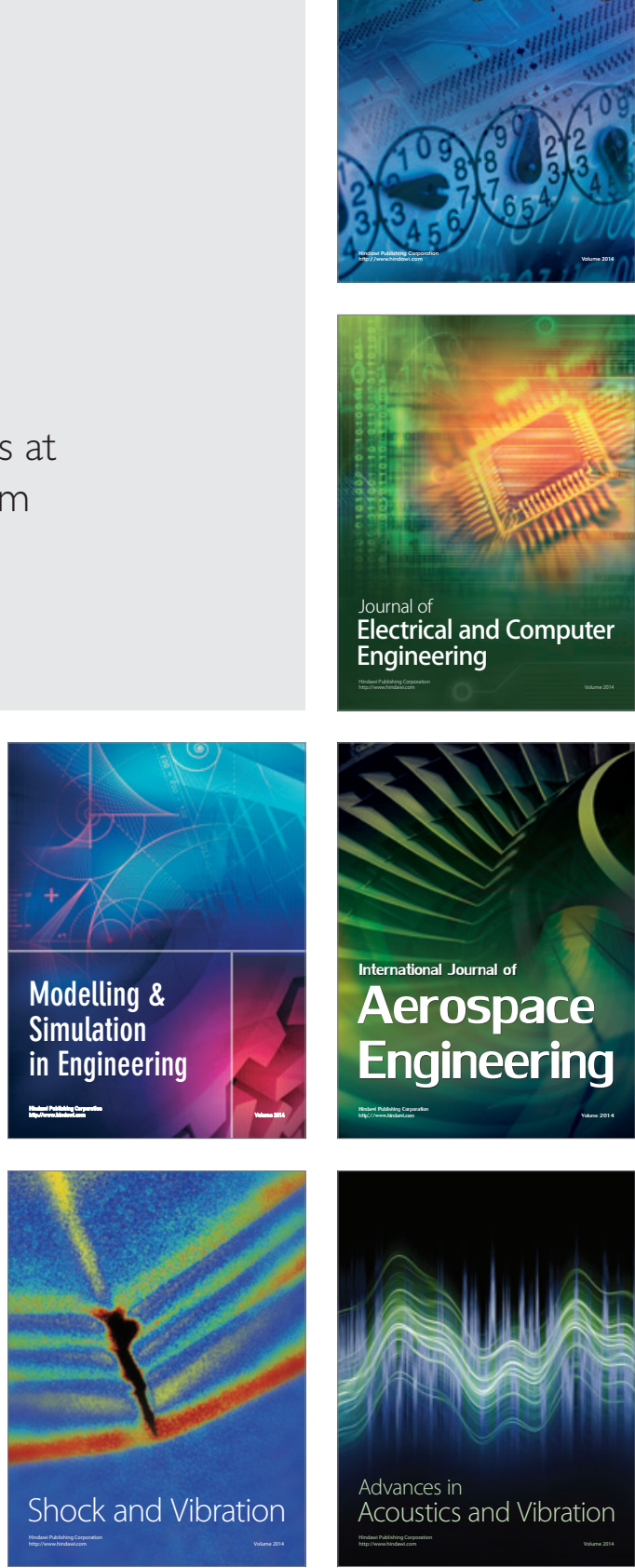